\title{
A ORIENTAÇÃO DA AÇÃO NO CONTEXTO DA TEORIA DE P. YA. GALPERIN COMO CONSTRUCTO DA PESQUISA EDUCACIONAL EM CIÊNCIAS: PERSPECTIVAS A PARTIR DO ESTADO DA QUESTÃO
}

Me. Alessandro Augusto de Barros Façanha Universidade Federal do Rio Grande do Norte

Dr. Isauro Beltrán Nuñez

Universidade Federal do Rio Grande do Norte

RESUMO: Este estudo investiga o estado da questão relativo à produção do conhecimento sobre a Orientação da ação de P. Ya. Galperin através de uma minuciosa revisão da produção científica em bases indexadas, bancos de teses e dissertações e eventos científicos para delimitar a natureza das produções na última década e a potencialidade do objeto como constructo de pesquisas no contexto do ensino das ciências. Como resultado se apresenta o atual estado da questão em torno da orientação da ação com evidências que a consolidam como objeto de pesquisa no campo conceitual, epistemológico, metodológico e didático aplicados ao ensino das ciências, contudo, indica possibilidades originais de investigação no contexto da orientação da ação relacionada às habilidades linguísticocognitivas, sobretudo em relação à orientação para leitura crítica no ensino das ciências.

PALAVRAS-CHAVE: Orientação da ação; Estado da questão; P. Ya. Galperin.

\section{THE GUIDANCE OF ACTION IN GALPERIN'S THEORY CONTEXT AS RESEARCH OBJECT IN SCIENCES: A REVIEW OF KNOWLEDGE}

\begin{abstract}
This study investigates the state of the issue about the production of knowledge for the Guidance of action through a thorough review of scientific production on research bases, thesis and dissertation banks and scientific events to delimit the nature of productions in the last decade and the potentiality of object as research construct in the context of science teaching. As a result, the present state of the issue is presented around the orientation of the action with
\end{abstract}

evidence that consolidates it as an object of research in the conceptual, epistemological, methodological and didactic field applied to the teaching of the sciences, however, it indicates original research possibilities in the context of orientation of the action related to the linguistic-cognitive abilities, especially in relation to the orientation for critical reading in the science teaching.

KEYWORDS: Guidance of action; research object in science; P. Ya. Galperin. 


\section{Educere "Educare \\ RevISTA DE Educacão}

Programa de Pós-Graduação em Educação - Universidade Estadual do Oeste do Paraná

\section{INTRODUÇÃO}

A pesquisa em educação, dada sua complexidade de investigação e elaboração de resultados consistentes com a práxis educativa necessita, mesmo a partir de recortes, conferir densidade em relação a delimitação do objeto em seu contexto, pois para além de um objeto em si, consiste em um aporte conceitual e metodológico que expressa a produção do conhecimento (LAROCCA e ROSSO, 2005).

Tal fato direciona o olhar do pesquisador para a inserção do objeto em um contexto que revele o estado do conhecimento em uma perspectiva espaço-tempo que possibilite delineamentos e recortes de investigação situados a partir de uma escala da produção de saberes.

Dessa forma, o estado da questão em relação ao objeto de investigação, tanto norteia quanto revela paradigmas que redimensionam a pesquisa em função de seu exercício investigativo para além do tempo presente deflagrando os aspectos atemporais dos fenômenos investigados além de definir as relações intrinsecas do objeto com o pesquisador em seu contexto (NOBREGA THERRIEN E THERRIEN, 2004).

No âmbito da pesquisa acadêmica a compreensão do estado da questão situa o pesquisador não apenas diante do objeto, mas defronte de sua problemática e das relações dialéticas que envolvem processos didáticos, metodológicos e paradigmáticos constituintes de um corpo teórico, no qual:

\footnotetext{
O estado da questão configura então o esclarecimento da posição do pesquisador e de seu objeto de estudo na elaboração de um texto narrativo, a concepção de ciência e a sua contribuição epistêmica no campo do conhecimento (NOBREGA THERRIEN E THERRIEN, 2004, p.5).
}

Confere assim um domínio conceitual que possibilita ao pesquisador além da competência técnica e instrumental da investigação, uma interpretação epistemológica coerente com um campo teórico e crítico que permite o 


\section{Educere "Educare \\ RevISTA DE Educacão}

Programa de Pós-Graduação em Educação - Universidade Estadual do Oeste do Paraná

desenvolvimento do argumento à luz da ciência e da produção do conhecimento científico (BELL, 2014).

Além disso, possibilita a compreensão do movimento existente em uma área do conhecimento, sua configuração, concepções teórico-metodológicas de suas produções bem como a análise crítica de seus paradigmas, tendências, recorrências e lacunas por ventura presentes no estado do conhecimento atribuído ao objeto (VOSGERAU e ROMANOWSKI, 2014).

Como constructo desta investigação se adota como objeto de análise a categoria da orientação da ação e sua intersecção no campo da pesquisa em ensino de Ciências, a fim de se compreender a evolução da produção científica nesta área do conhecimento. A intenção do estudo surge da necessidade de se mapear tal constructo como balizador para investigações que envolvem a orientação da ação no campo da pesquisa relacionada ao ensino das Ciências e seus desdobramentos. Assim, em função das questões problematizadoras que envolvem esse campo de investigação e com o objetivo de revisar o estado da questão da orientação da ação se partiu dos seguintes questionamentos iniciais: Como se situa a produção científica em torno da orientação da ação na perspectiva do ensino das Ciências? Qual o estado do conhecimento científico produzido ao longo da ultima década acerca da orientação da ação no campo das pesquisas do ensino das Ciências e seus desdobramentos? Quais as intersecções entre a orientação da ação de P. Ya. Galperin e as demandas relacionadas às pesquisas no ensino das Ciências? Quais as possibilidades para futuras investigações em termos de relevância e originalidade no contexto da orientação da ação?

\section{Aspectos conceituais da orientação da ação.}

A orientação da ação consiste em um esquema racional e consciente de como se deve fazer para se assimilar um dado conceito/habilidade que encerra em si, através de um modelo da ação um arcabouço operacional de ações 


\section{Educere "Educare \\ RevISTA de EduCACÃo}

Programa de Pós-Graduação em Educação - Universidade Estadual do Oeste do Paraná

concretas que constituem um processo de abstração do real por meio da atividade (LURIA; LEONTIEV, 2004).

A atividade corresponde a uma premissa teórica do materialismo histórico dialético que no sentido psicológico e cognoscitivo constitui as relações entre o sujeito-objeto caracterizadas como um conjunto da práxis projetada pela psique que dá sentido aos objetos e permite sua internalização (LEONTIEV, 1981).

Como destaca Talízina (1988), o conceito de atividade congrega um conjunto de processos sintetizadores de uma atitude vital do homem enquanto ser social, histórico e ativo em relação ao meio que o cerca. Reafirmando esse caráter, porém na perspectiva dos processos cognoscitivos de assimilação dos conceitos e habilidades reside a orientação da ação de P. Ya. Galperin, que se define como um domínio de ações da dimensão psíquica e da prática que permitem a regulação racional da atividade internalizada.

Como marco teórico investigativo o conceito de orientação da ação se encontra inserido na Teoria da Formação Planejada das Ações Mentais de P. Ya. Galperin, onde se caracteriza como uma etapa diretiva do processo de ensinoaprendizagem, na qual se configuram, em termos do objeto a ser internalizado, as operações que devem ser executadas e controladas para a aquisição conceitual ou formação de uma habilidade (GALPERIN, 1986).

Portanto, a orientação da ação se constitui enquanto objeto de pesquisa como uma variável multifatorial e inserida em diversos campos do conhecimento, pois, se situa como pressuposto do desenvolvimento cognoscitivo, como premissa de aprendizagem e também como um subsistema relacionado à atividade e a formação de funções psíquicas superiores. Congregando essa multiplicidade relaciona-se diretamente na assimilação e no desenvolvimento de habilidades e conceitos mediatizados a partir de uma sequência de atividades sistemáticas e inter-relacionadas que envolvem o professor e os alunos em uma 


\section{Educere "Educare \\ RevISTA DE Educacão}

Programa de Pós-Graduação em Educação - Universidade Estadual do Oeste do Paraná

dimensão que compreende a assimilação, a atividade consciente e a orientação (NUÑEZ, 2009).

No âmbito deste estudo, que visa investigar o estado da questão da orientação da ação com o intuito de balizar pesquisas futuras no campo do ensino a partir da compreensão de que essas categorias se inserem no escopo das atividades humanas mediatizadas, a orientação da ação passa a se constituir como um conhecimento profissional desejável para futuros docentes, uma vez que, no campo das didáticas a atividade de orientação subsidia sistemas estruturantes do ensino, aprendizagem e demais campos epistemológicos.

Dessa forma, no âmbito das pesquisas em educação e no ensino, desvelar a orientação da ação enquanto construto de pesquisa confere maior amplitude na pesquisa em torno de um redimensionamento epistemológico, além de anunciar as derivações relativas ao objeto, sua natureza, contextualização e conceituação em função da produção do conhecimento atual e balizar caminhos para a produção futura em função dos eventuais hiatos e necessidades.

\section{A orientação da ação como uma categoria do desenvolvimento cognoscitivo.}

O desenvolvimento cognoscitivo é uma categoria presente nas bases do desenvolvimento da Psicologia Histórico-Cultural que teve como precursor Lev S. Vigotsky, e grandes expoentes derivados desses estudos, como Leontiev e Luria, os quais juntamente com Vigotsky compunham a chamada Troika, que atuou no desenvolvimento de uma teoria psico-cognitiva acerca do desenvolvimento e aprendizagem; além de outros teóricos que de forma específica aprofundaram os estudos iniciais com enfoques distintos, como D. B. Elkonin, A. V. Zaporozhets, P. Ya. Galperin, V. V. Davídov, L. V. Zankov, N. F. Talizina, V. S. Mujina, L. I. Bozhovich, A. Petrovski e P. I. Zinchenko; que em conjunto formam um escopo teórico profundo acerca da denominada didática desenvolvimental, (TOASSA, 2016; PUENTES e LONGAREZI, 2013; LIBÂNEO e FREITAS, 2013). 


\section{Educere Educare \\ RevISTA DE EducAcão}

Programa de Pós-Graduação em Educação - Universidade Estadual do Oeste do Paraná

Independente das especificidades de cada teórico e seus respectivos campos de estudo, em conjunto compõem um corpo de estudiosos de orientação marxista que discutem e teorizam, à luz do materialismo histórico dialético, as bases da compreensão do desenvolvimento humano, da psicogênese da aprendizagem e da influência histórico-cultural envolvidas nos processos de formação cognitiva.

Ressalta-se que o processo primordial que embasa e fundamenta a abordagem investigativa a partir desta concepção busca, em essência, o estudo da formação de um desenvolvimento psíquico que subsidia a internalização conceitual e a formação de representações mentais superiores centradas na mediação cultural com enfoque na linguagem e na abstração objetal a partir da mediatização em torno da atividade cujo princípio recapitula, diferentemente dos demais animais, estágios de desenvolvimento ontogenético (LEONTIEV, 1978).

(...) o desenvolvimento filogenético dos animais ocorre "pela consolidação das mudanças na sua organização biológica", no caso do ser humano o seu desenvolvimento histórico se consolida pelos "objetos materiais e em fenômenos ideais". Desse modo, o desenvolvimento humano se dá pelos processos de assimilação ou apropriação do desenvolvimento evolutivo de gerações anteriores. (...) para "se apropriar" dos objetos ou fenômenos é necessário executar uma atividade adequada ao conteúdo no objeto ou no fenômeno dado, mas as ações e operações estão relacionadas com o objeto (LEONTIEV, 1978, p. 142).

Tomando a atividade sob a categoria da ação humana como defende Martins e Moser (2012), o desenvolvimento do psiquismo e por consequência das funções mentais superiores demanda uma ação organizada, planejada e executada com orientação e controle, uma vez que pressupõe a formação de estruturas psíquicas complexas que guardam interdependência entre o objeto, a cultura e as representações semióticas.

Sendo assim, a consciência é parte desse processo sendo que sua formação se dá através de um fluxo entre a materialidade e a abstração a partir da mediação das relações histórico-culturais que pressupõem uma unidade dialética entre a consciência e a atividade a partir da qual: 


\section{Educere "Educare \\ ReVISTA de EduCACÃo}

Programa de Pós-Graduação em Educação - Universidade Estadual do Oeste do Paraná

É a análise da atividade que constitui o ponto decisivo e o método principal do conhecimento científico do reflexo psíquico da consciência. Nas formas da consciência social está a análise do cotidiano, da sociedade e das formas de produção das relações sociais; no estudo da pique individual está a análise da atividade dos indivíduos nas condições sociais dadas e nas circunstâncias concretas que lhes é dado (LEONTIEV, 1978, p.90)

Tais premissas inauguram uma nova perspectiva de entendimento do desenvolvimento humano uma vez que refutam as ideias defendidas pela teoria idealista clássica e constituem um novo fundamento epistemológico que defende, sobretudo a partir de estudos de Luria e Vigotsky, a tese de que as construções cognitivas derivam da atividade humana em função de variáveis relacionadas ao comportamento, a regulação de processos de internalização e mediatização através da cultura, da linguagem e da própria ação humana (LURIA, 1992; VIGOTSKY, 2003).

Além disso, do ponto de vista epistemológico, não só inauguram uma nova concepção de compreender os fenômenos psico-cognitvos como rompem com um modelo dicotômico de ensino (estímulo)/aprendizagem, incorporando a dinâmica sociocultural ao processo e ampliando o desenvolvimento humano aliado à linguagem, comportamento e relações sociais a partir de uma conceituação ampla das funções mentais superiores.

Nesse sentido, realizando a intersecção do objeto da orientação a partir dos pressupostos teóricos de P. Ya. Galperin, o enfoque específico da orientação da ação sustenta o princípio de que é possivel, através da atividade planejada, se constituírem processos mentais e assimilações conceituais instituídas a partir da atividade de forma tal que a partir de um processo de operações em torno de um dado conceito/habilidade se viabilize sua internalização mediante a formação de representações mentais que conferem um domínio de ações na dimensão psíquica e prática que permitam a regulação da atividade internalizada (TALÍZINA, 2001). 


\section{Educere "Educare \\ RevISTA de EduCACÃo}

Programa de Pós-Graduação em Educação - Universidade Estadual do Oeste do Paraná

O processo de internalização da atividade externa em interna é concebido como um ciclo cognoscitivo, no qual se destacam momentos funcionais orientação, execução e controle - que, se não podem ser considerados de forma linear, podem ser separados, metodologicamente, para análise (GALPERIN, 1986).

Neste sentido, em termos dos aspectos relacionados ao desenvolvimento, amplificando os estudos iniciais de Leontiev e de Vigotsky no que tange o desenvolvimento cognoscitivo, e ampliando a perspectiva da aprendizagem como um processo de reconfigurações da atividade orientadora da ação, onde o núcleo se estrutura a partir do processo de internalização da atividade objetal (material) em interna (abstrata), credenciam-se os estudos de P. Ya. Galperin, como um marco da formação de conceitos/habilidades a partir da assunção de que a etapa orientadora da ação é o condutor do desenvolvimento psíquico superior, sendo assim, a partir de um esboço do estado da questão desta categoria conceitual é possivel compreender a produção do conhecimento atual e traçar novas possibilidades de investigação.

\section{A orientação da ação como objeto de pesquisa: a elaboração do estado da questão.}

Distinguir entre a determinação do estado da questão da pesquisa e a elaboração de seu estado de arte evidencia no campo das categorias teóricas do trabalho de pesquisa um meio para compreender as referências e o estado do conhecimento a fim de se ampliar a temática e a configuração dos argumentos em torno do objeto, além disso, reorganiza os dados relativos ao objeto e aguça a seleção criteriosa da produção científica, pois pressupõe o confronto com a proposta de investigação na qual se intenta pesquisar (NÓBREGA-THERRIEN e THERRIEN, 2004, 2010; GALVÃO, 2010; BORBA e VALDEMARIN, 2010).

Nessa perspectiva o estado da questão a ser construído neste percurso visa, para além da pesquisa acadêmica e seus desdobramentos, a consolidação de um constructo investigativo que possibilite, a partir da lógica da pesquisa 


\section{Educere "Educare \\ ReVISTA DE EduCACÃo}

Programa de Pós-Graduação em Educação - Universidade Estadual do Oeste do Paraná

contribuir para a consolidação de um campo didático, metodológico e epistemológico, que articule as concepções e intenções de uma tese e ao mesmo tempo construa as respostas sociais esperadas para solucionar problemas relacionados ao objeto e suas implicações.

Com esse intuito, se objetiva ampliar o olhar sobre o atual estado do conhecimento produzido sobre a orientação da ação para a habilidade da leitura crítica no âmbito dos conhecimentos profissionais necessários para o ensino de Química a fim de refletir-se sobre a construção de um objeto de pesquisa que delimite essa orientação em função das categorias relacionadas ao contexto da profissionalização e formação de professores de Ciências/Química.

Tais assertivas e critérios de análise do objeto tomaram como base a lógica defendida como estrutura concebida nos estudos cujo propósito é imergir no estado epistemológico da questão, que:

(...) vem subsidiar, portanto, todo o processo de investigação, ajudando a redefinir os objetivos, auxiliando no planejamento do campo teóricometodológico, contribuindo na identificação das categorias teóricas, nas discussões e análises dos dados e, finalmente, mostrando, na conclusão da pesquisa, de forma explicita ou implícita, a sua contribuição para a construção do conhecimento (NOBREGA-THERRIEN; THERRIEN, 2010, p. 220).

Dessa forma, partindo do nivel generalizado para o específico se dimensionou o objeto em função de categorias importantes para um retrato de seu estado da questão no momento atual contemplando assim critérios importantes para o desenho de trabalhos e teses que dimensionam originalidade e novidade à pesquisa, pois se desvela o objeto em dimensões amplas e de densidade conceitual e metodológica.

Assim, a partir de uma ampla base de dados nacionais, internacionais e de eventos relevantes para a didática e o ensino das ciências se desenhou uma estratégia metodológica de levantamento de produções e publicações indexadas disponiveis no interstício dos últimos anos em sítios de pesquisas e dados como 


\section{Educere "Educare \\ ReVISTA DE EduCACÃo}

Programa de Pós-Graduação em Educação - Universidade Estadual do Oeste do Paraná

o portal de periódicos da Capes, o Science Education Internacional, a base de editores Taylor and Francis e a Dialnet, pois englobam em conjunto publicações de natureza nacionais, internacionais e de eventos validados pela comunidade científica.

Como estratégia de busca se utilizou de descritores genéricos e específicos que compõem o quadro de banco de palavras-chave que convergissem o objeto de pesquisa para o campo da investigação nas áreas da orientação da ação, base orientadora e formação de conceitos, orientação da ação no âmbito da teoria de P. Ya. Galperin, bem como em alusão à sua inserção no contexto da formação de habilidades cognitivas a partir da Teoria da Atividade.

Estima-se que assim que foi possivel dimensionar o objeto da pesquisa a orientação da ação - em suas múltiplas perspectivas estruturais e conceituais abrangendo ao estado da questão da pesquisa a dimensão da produção de conhecimentos em seus níveis epistemológicos, gnosiológicos e cognoscitivos.

\section{Os dados revelados e seu contexto: o estado da questão e} possiveis interpretações para o campo epistemológico da didática das ciências.

Conforme explicitado no campo do percurso de elaboração do estado da questão em análise, uma das plataformas de mapeamento foi o banco de teses e dissertações por se creditar a esse tipo de produção um percurso de construção do conhecimento válido para mensuração do avanço conceitual e sua relação intrínseca com os programas de pós-graduação e as intensas atividades de grupos de pesquisa cujos trabalhos conferem validade e credibilidade na construção epistemológica do saber, desta feita os quadros 1 e 2 trazem estas dimensões em destaque: 


\section{Educere Educare \\ RevISTA De EduCAC̄̃o}

Programa de Pós-Graduação em Educação - Universidade Estadual do Oeste do Paraná

Quadro 1: Produções de Dissertações que envolvem a categoria da orientação da ação, seu local de produção e ano de publicação.

\begin{tabular}{|c|c|c|}
\hline Área temática da produção & Instituição ${ }^{\mathbf{i}}$ & $\begin{array}{l}\text { Ano da } \\
\text { produção }\end{array}$ \\
\hline 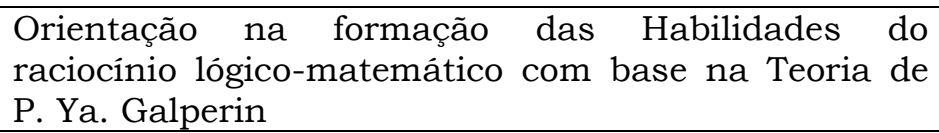 & \multirow{4}{*}{ UERR } & \multirow{7}{*}{2015} \\
\hline $\begin{array}{l}\text { Orientação na formação dos conceitos envolvendo a } \\
\text { Lógica Matemática a partir da teoria de P. Ya. } \\
\text { Galperin }\end{array}$ & & \\
\hline $\begin{array}{lll}\text { Orientação para o ensino problematizador } & \text { no } \\
\text { processo de formação e assimilação conceitos } & \text { na } \\
\text { perspectiva da teoria de P. Ya. Galperin } & & \\
\end{array}$ & & \\
\hline $\begin{array}{l}\text { Orientação na formação dos conceitos envolvendo } \\
\text { Derivadas a partir da teoria de P. Ya. Galperin }\end{array}$ & & \\
\hline 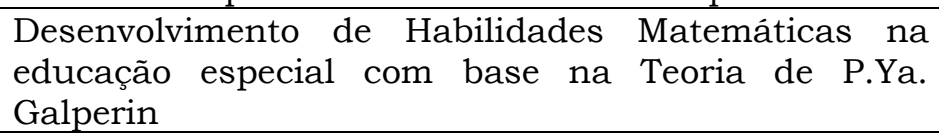 & IFES & \\
\hline $\begin{array}{l}\text { Orientação para Ensino de Álgebra a partir da Teoria } \\
\text { de P.Ya. Galperin }\end{array}$ & UNIUBE & \\
\hline $\begin{array}{l}\text { Orientação na formação de Habilidades cognitivas } \\
\text { para o Ensino de Química com base na Teoria de P. } \\
\text { Ya. Galperin }\end{array}$ & UFRN & \\
\hline $\begin{array}{l}\text { Orientação na Aprendizagem de conceitos } \\
\text { matemáticos a partir da Teoria de P.Ya. Galperin }\end{array}$ & \multirow{5}{*}{ UERR } & \multirow{6}{*}{2014} \\
\hline $\begin{array}{l}\text { Orientação para a Problematização na perspectiva da } \\
\text { Teoria de P. Ya. Galperin }\end{array}$ & & \\
\hline $\begin{array}{l}\text { Orientação na formação de Conceitos da Cinemática } \\
\text { na perspectiva da Teoria de P. Ya. Galperin }\end{array}$ & & \\
\hline $\begin{array}{l}\text { Orientação na formação de conceitos de ciências na } \\
\text { perspectiva da Teoria de P. Ya. Galperin }\end{array}$ & & \\
\hline $\begin{array}{l}\text { Educação científica a partir da Teoria Histórico- } \\
\text { cultural }\end{array}$ & & \\
\hline $\begin{array}{l}\text { Orientação para Ensino de língua portuguesa na } \\
\text { perspectiva da Teoria de P. Ya. Galperin }\end{array}$ & UNIUBE & \\
\hline 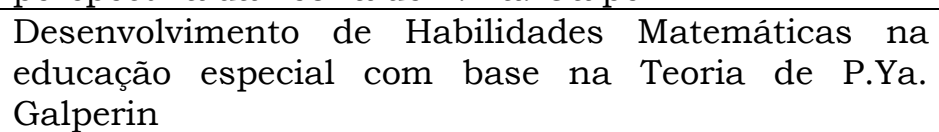 & UTFPR & 2013 \\
\hline
\end{tabular}

(Fonte: autores do estudo)

Quadro 2: Produções de Teses que envolvem a categoria da orientação da ação, seu local de produção e ano de publicação.

\begin{tabular}{|c|c|c|}
\hline Área temática da produção & Local/Instituiçãoii & $\begin{array}{c}\text { Ano da } \\
\text { produção }\end{array}$ \\
\hline $\begin{array}{c}\text { Orientação para a formação de } \\
\text { Competências da linguagem a partir } \\
\text { da Teoria de P. Ya. Galperin }\end{array}$ & UFU & \multirow{2}{*}{2015} \\
\hline $\begin{array}{c}\text { Estudos da Atenção no campo do } \\
\text { Desenvolvimento a partir da } \\
\text { perspectiva Histórico-cultural }\end{array}$ & USP & \\
\hline
\end{tabular}




\section{Educere "Educare \\ RevISTA De EduCAC̄̃̃o}

Programa de Pós-Graduação em Educação - Universidade Estadual do Oeste do Paraná

\begin{tabular}{|c|c|c|}
\hline $\begin{array}{l}\text { Orientação para a formação de } \\
\text { habilidades linguístico-cognitivas a } \\
\text { partir da Teoria de P. Ya. Galperin }\end{array}$ & \multirow{3}{*}{ UFRN } & \multirow{5}{*}{2014} \\
\hline $\begin{array}{c}\text { Orientação para aprendizagem de } \\
\text { História a partir da perspectiva } \\
\text { Histórico-cultural }\end{array}$ & & \\
\hline $\begin{array}{c}\text { Pesquisa sobre Profissionalização } \\
\text { docente na perspectiva da Teoria da } \\
\text { Atividade }\end{array}$ & & \\
\hline $\begin{array}{c}\text { Orientação para a formação de } \\
\text { conceitos de Físico-química a partir } \\
\text { da Teoria de P. Ya. Galperin }\end{array}$ & UFRPE & \\
\hline $\begin{array}{c}\text { Orientação para aprendizagem de } \\
\text { conceitos matemáticos a partir da } \\
\text { Teoria de P. Ya. Galperin }\end{array}$ & UFPB & \\
\hline $\begin{array}{c}\text { Orientação para a formação de } \\
\text { habilidades cognitivas no Ensino de } \\
\text { Química a partir da Teoria de P. Ya. } \\
\text { Galperin }\end{array}$ & \multirow[t]{2}{*}{ UFRN } & \multirow[t]{2}{*}{2013} \\
\hline $\begin{array}{c}\text { Pesquisa sobre Profissionalização } \\
\text { docente na perspectiva da Teoria da } \\
\text { Atividade }\end{array}$ & & \\
\hline
\end{tabular}

(Fonte: autores do estudo)

Percebe-se por esse esboço que a temática inerente à orientação da ação

é um campo recorrente quando das pesquisas que envolvem as categorias da formação de conceitos e habilidades bem como associadas ao contexto da aprendizagem. Tais prerrogativas encontram subsidio a partir da perspectiva da psicologia e da didática desenvolvimental e sua intensa contribuição no campo do paradigma histórico-cultural a partir de contribuições para uma nova concepção acerca do processo de ensino-aprendizagem e paradigmas de educação à luz do desenvolvimento humano (SILVA FAQUIM e PUENTES, 2013).

Contrapondo-se ao modelo empirista-dedutivista e ao paradigma condutista de aprendizado, onde a transmissão/recepção é privilegiada, estas pesquisas corroboram para a consolidação do paradigma desenvolvimental onde o cerne do desenvolvimento reside na perspectiva cognoscitiva subsidiada pela concepção histórico-cultural a qual tem na atividade sua premissa estrutural e onde se percebe que a orientação da ação se constitui na base orientadora do aprendizado. Observar estes pressupostos em uma série de pesquisas 


\section{Educere Educare \\ RevISTA DE Educacão}

Programa de Pós-Graduação em Educação - Universidade Estadual do Oeste do Paraná

defendidas a partir de um corpo de dissertações e teses aponta para um novo olhar sobre as didáticas, onde:

Las escuelas deben ser generadoras de cambios en el mejoramiento y la calidad del proceso de enseñanza aprendizaje. (...) conciben este proceso como un todo integrado entre lo cognitivo, lo afectivo, lo instructivo y lo educativo, cuyo centro debe ser la actividad y la comunicación del estudiante (Toruncha e Cruz, 2017, p.244).

Além disso, em relação aos aspectos da natureza do conhecimento científico em função do estado da questão, oportuniza uma leitura acerca das convergências em torno do conceito da orientação da ação como um marco importante que intersecciona os olhares em torno da formação de conceitos e habilidades, o processo de desenvolvimento a partir da aprendizagem orientada e as relações entre a orientação e as pesquisas no campo da didática das ciências e matemática.

O mapeamento em periódicos internacionais de relevância no campo da didática, dos estudos que envolvem aprendizagem, estudos da psicologia relacionados ao desenvolvimento e revistas específicas de educação em ciências/química, trouxe resultados importantes para ampliar a elaboração do estado da questão em torno da orientação da ação e dos desdobramentos conceituais envolvidos, como a Teoria da formação planejada por etapas das ações mentais e dos conceitos, desenvolvida por P. Ya. Galperin, as concepções histórico-culturais do desenvolvimento além da Teoria da Atividade de Leontiev, dessa forma, agrupando-os no quadro 3 a seguir é possivel perceber essas nuances.

Quadro 3: Produções em periódicos internacionais distribuídos por área de conhecimento e ano de publicação.

\begin{tabular}{|c|c|l|c|}
\hline Nome do Periódico & $\begin{array}{l}\text { Quantidade } \\
\text { de artigos } \\
\text { publicados }\end{array}$ & \multicolumn{1}{|c|}{ Área de conhecimento } & Ano \\
\hline \multirow{2}{*}{ Mind, Culture, and Activity } & 3 & $\begin{array}{l}\text { Orientação da Ação na na } \\
\text { perspectiva da Teoria da P.Ya. } \\
\text { Galperin }\end{array}$ & 2010 \\
\cline { 2 - 4 } & 2 & Teoria da Atividade & 2011 \\
\hline
\end{tabular}




\section{Educere" Educare \\ RevisTa de Educacẽo}

Programa de Pós-Graduação em Educação - Universidade Estadual do Oeste do Paraná

\begin{tabular}{|c|c|c|c|}
\hline & 2 & $\begin{array}{lr}\text { Concepções do } & \text { paradigma } \\
\text { Histórico-cultural } & \text { em } \\
\text { educação } & \\
\end{array}$ & 2014 \\
\hline $\begin{array}{l}\text { Journal of Russian and East } \\
\text { European Psychology }\end{array}$ & 2 & $\begin{array}{l}\text { Orientação da Ação na } \\
\text { perspectiva da Teoria da P.Ya. } \\
\text { Galperin }\end{array}$ & 2014 \\
\hline $\begin{array}{lcc}\text { International Journal } & \text { of } \\
\text { Pedagogical Innovations } & \end{array}$ & 1 & Teoria da atividade & 2013 \\
\hline \multirow[t]{2}{*}{$\begin{array}{l}\text { Psychology in Russia: state } \\
\text { of art }\end{array}$} & 3 & $\begin{array}{l}\text { Orientação da Ação na } \\
\text { perspectiva da Teoria da P.Ya. } \\
\text { Galperin }\end{array}$ & 2010 \\
\hline & 3 & $\begin{array}{l}\text { Orientação da Ação na } \\
\text { perspectiva da Teoria da P.Ya. } \\
\text { Galperin }\end{array}$ & 2014 \\
\hline $\begin{array}{l}\text { Journal of Russian and East } \\
\text { European Psychology }\end{array}$ & 4 & $\begin{array}{lrr}\text { Concepções do } & \text { paradigma } \\
\text { Histórico-cultural } & \text { em } \\
\text { educação } & \end{array}$ & 2014 \\
\hline $\begin{array}{l}\text { Chemistry Education } \\
\text { Research and Practice }\end{array}$ & 1 & $\begin{array}{l}\text { Orientação da Ação na } \\
\text { perspectiva da Teoria da P.Ya. } \\
\text { Galperin }\end{array}$ & 2009 \\
\hline $\begin{array}{l}\text { The Moscow University } \\
\text { Herald/ Series Psychology }\end{array}$ & 2 & $\begin{array}{l}\text { Orientação da Ação na } \\
\text { perspectiva da Teoria da P.Ya. } \\
\text { Galperin }\end{array}$ & 2012 \\
\hline \multirow{2}{*}{$\begin{array}{l}\text { Innovations in Education } \\
\text { and Teaching International }\end{array}$} & 2 & Teoria da Atividade & \multirow{2}{*}{2012} \\
\hline & 1 & $\begin{array}{lr}\text { Concepções do } & \text { paradigma } \\
\text { Histórico-cultural } & \text { em } \\
\text { educação } & \\
\end{array}$ & \\
\hline
\end{tabular}

(Fonte: autores do estudo)

Pelas produções se observa uma forte correlação entre o paradigma Histórico-cultural em suas diversas concepções teóricas acerca do desenvolvimento, aprendizagem e didática, que foram categorizadas em função da elaboração do estado da questão a partir da: Orientação da Ação na perspectiva da Teoria da P.Ya. Galperin, Concepções do paradigma Históricocultural em educação e Teoria da Atividade. Tal categorização em função de estabelecer uma classificação mais didática para agrupar os trabalhos em função do arcabouço teórico-metodológico envolvido nos estudos que se diversificavam ora em relação ao enfoque com o conceito de orientação da ação e aprendizagem, ora com os aspectos gerais da didática desenvolvimental e educação, sobretudo com enfoque as ideias de Lev Vigotsky e o desenvolvimento. 


\section{Educere "Educare \\ RevISTA de EduCACÃo}

Programa de Pós-Graduação em Educação - Universidade Estadual do Oeste do Paraná

Sendo assim em função do estado da questão em si, observam-se fortes influências dos trabalhos da Escola de Jarkov nas pesquisas de natureza educacional tanto no âmbito dos países da antiga URSS como nos grupos de pesquisadores europeus demonstrando, além da influência geopolítica do pensamento oriental de desenvolvimento e ensino, os desdobramentos dessa tradição educacional no contexto das didáticas.

Tais evidências, apesar de algumas distorções conceituais próprias das dificuldades de tradução dos originais são percebidas em relação aos estudos brasileiros, como foi destacado no mapeamento das produções acadêmicas nacionais, como no próprio contexto da pedagogia brasileira (MOLL, 2002; REGO, 2013), sobretudo pela penetração das ideias precursoras de Vygotsky e Leontiev, como exemplificado no corpo de tais produções originadas em grupos de pesquisas em algumas universidades e programas de pós-graduação, os quais desenvolvem uma produção de conhecimento considerável a partir do paradigma histórico-cultural, do desenvolvimento cognoscitivo da atividade e nas pesquisas sobre formação de conceitos e habilidades.

Assim, a luz desse inventariado é possivel se compreender que as habilidades linguístico-cognitivas em função da orientação da ação se constituem como um grande eixo norteador na produção do conhecimento em ciências e matemática, porém, especificamente sobre a orientação da ação no contexto da leitura crítica ainda não se revela uma sólida produção. Assim, se denota que este referencial ainda se revela como um conhecimento a ser desvelado com originalidade e relevância para a pesquisa acadêmica, a formação de habilidades linguístico-cognitivas e o conhecimento didático das ciências no campo da epistemologia da aprendizagem.

Nessa perspectiva, entretanto, a fim de relacionar o estado da questão em uma abrangência conceitual e metodológica também optou-se por realizar uma imersão nessa produção de conhecimento no campo da prática docente e das atividades práticas das ciências da educação brasileira, realizando-se um 


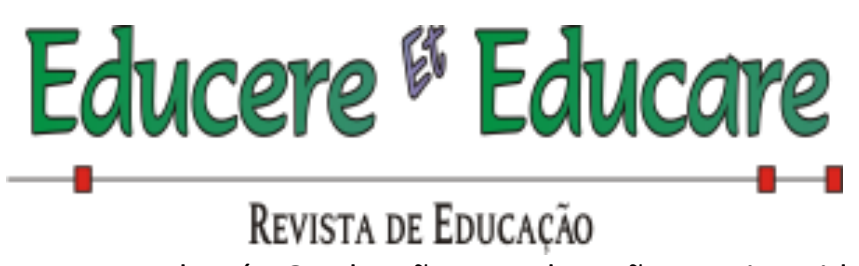

Programa de Pós-Graduação em Educação - Universidade Estadual do Oeste do Paraná

mapeamento dessa produção científica no âmbito dos trabalhos apresentados em eventos nacionais de recorrência de temáticas de ensino de ciências onde se encontra um sítio de produção de pesquisadores cujas atividades englobam a escola, os grupos específicos de educação científica e os projetos em parceria da universidade com as escolas de educação básica.

Nesse aspecto, se elegeu como referência as produções situadas nos anais do Encontro Nacional de Pesquisa em Educação em Ciências (ENPEC), pois se trata de um evento chancelado pela Associação Brasileira de Pesquisa em Educação em Ciências (ABRAPEC). A respeito dessas produções, como a edição é bianual elencou-se os trabalhos da última década, conforme ilustrado no quadro 4 a seguir:

Quadro 4: Conhecimento produzido na última década do ENPEC - Encontro Nacional de Pesquisa em Educação em Ciências.

\begin{tabular}{|c|c|c|}
\hline Ano & Dimensão conceitual e teórica do trabalho & $\begin{array}{l}\text { Número de } \\
\text { produções }\end{array}$ \\
\hline \multirow{4}{*}{2005} & Orientação da Ação na perspectiva da Teoria da P.Ya. Galperin & 1 \\
\hline & Concepções do paradigma Histórico-cultural em educação & 3 \\
\hline & $\begin{array}{l}\text { Formação de conceitos e Habilidades cognitivas a partir da teoria da } \\
\text { P.Ya. Galperin }\end{array}$ & 2 \\
\hline & $\begin{array}{l}\text { Teoria da Atividade } \\
\end{array}$ & Nenhum \\
\hline \multirow{4}{*}{2007} & Orientação da Ação na perspectiva da Teoria da P.Ya. Galperin & 1 \\
\hline & Concepções do paradigma Histórico-cultural em educação & 3 \\
\hline & $\begin{array}{l}\text { Formação de conceitos e Habilidades cognitivas a partir da teoria da } \\
\text { P.Ya. Galperin }\end{array}$ & 1 \\
\hline & Teoria da Atividade & Nenhum \\
\hline \multirow{4}{*}{2009} & Concepções do paradigma Histórico-cultural em educação & 3 \\
\hline & Orientação da Ação na perspectiva da Teoria da P.Ya. Galperin & Nenhum \\
\hline & $\begin{array}{l}\text { Formação de conceitos e Habilidades cognitivas a partir da teoria da } \\
\text { P.Ya. Galperin }\end{array}$ & 1 \\
\hline & $\begin{array}{l}\text { Teoria da Atividade } \\
\end{array}$ & Nenhum \\
\hline \multirow{4}{*}{2011} & Orientação da Ação na perspectiva da Teoria da P.Ya. Galperin & 3 \\
\hline & Concepções do paradigma Histórico-cultural em educação & 2 \\
\hline & $\begin{array}{l}\text { Formação de conceitos e Habilidades cognitivas a partir da teoria da } \\
\text { P.Ya. Galperin }\end{array}$ & 3 \\
\hline & Teoria da Atividade & 6 \\
\hline & Orientação da Ação na perspectiva da Teoria da P.Ya. Galperin & 2 \\
\hline
\end{tabular}

Revista Educere Et Educare, Vol. 13, N. 30, nov./dez. 2018. Ahead of Print. DOI: 10.17648/educare.v13i30.18804 


\section{Educere Educare \\ ReVISTA DE EduCAC̄̃o}

Programa de Pós-Graduação em Educação - Universidade Estadual do Oeste do Paraná

\begin{tabular}{|c|c|c|}
\hline \multirow{4}{*}{2013} & Concepções do paradigma Histórico-cultural em educação & 1 \\
\cline { 2 - 3 } & $\begin{array}{c}\text { Formação de conceitos e Habilidades cognitivas a partir da teoria da } \\
\text { P.Ya. Galperin }\end{array}$ & 5 \\
\cline { 2 - 3 } & Teoria da Atividade & 1 \\
\hline \multirow{3}{*}{2015} & Orientação da Ação na perspectiva da Teoria da P.Ya. Galperin & 1 \\
\cline { 2 - 3 } & Formação de conceitos e Habilidades cognitivas a partir da teoria da \\
\cline { 2 - 3 } & P.Ya. Galperin & Nenhum \\
\cline { 2 - 3 } & Teoria da Atividade & Nenhum \\
\hline
\end{tabular}

(Fonte: autores do estudo)

Os resultados desse mapeamento específico foram bastante contundentes para a compreensão do espectro do estado da questão em função das produções que além do campo epistemológico que envolvem a ordem pragmática do conhecimento uma vez que se configuram como um corpo conceitual da ordem e da razão instrumental e procedimental da profissão docente e de futuros docentes, pois engloba em sua natureza além da produção de grupos de pesquisa e de pós-graduação, a geração do conhecimento em nível das pesquisas de iniciação científica, dos programas de iniciação à docência e dos resultados preliminares de estudos desenvolvidos no âmbito da escola.

Nesse sentido vale destacar os trabalhos que envolvem a orientação da ação tanto ao nível teórico, como na formação de conceitos e habilidades que aparecem em quase todas as edições do evento demonstrando assim, enquanto estado da questão:

(...) a reflexão sobre o que esta sendo pesquisado na atualidade e o enfoque priorizado pelos estudos encontrados com relação ao nosso tema de interesse. Assim, podemos relacionar os achados com a nossa intenção de pesquisa, identificando pontos semelhantes, convergentes e divergentes, encontrados nos estudos mapeados, identificando perspectivas plurais de teóricos sobre o tema, e, ainda, as contribuições que a nossa proposta investigativa poderá trazer para essa área do conhecimento (SILVEIRA e NÓBREGA-THERRIEN 2011, p. 225).

Dessa forma se chega ao estado da questão em si, amplificando sua dimensão enquanto objeto em discussão e tomando uma dimensão de estado de 


\title{
Educere Educare \\ RevISTA DE Educacão
}

Programa de Pós-Graduação em Educação - Universidade Estadual do Oeste do Paraná

conhecimento norteador e referência de investigação, pois como explica Bell (2014), elabora-se como campo de pesquisa a partir de uma configuração de domínio conceitual, de revisão e de base epistemológica garantindo um refinamento e uma reelaboração a partir de uma discussão crítica em torno do objeto contumaz ao processo de construção do amplo estado da questão da pesquisa.

\begin{abstract}
São esses atributos, imbricados no domínio conceitual e da literatura, que propelem a elaboração da argumentação que irá moldar o desenvolvimento do estado da questão. (...) requer uma compreensão ampla da problemática em foco fundada nos registros dos achados científicos e nas suas bases teórico-metodológicas acerca da temática e, decorrente desse mergulho, requer igualmente a perspectiva de contribuição do próprio estudante/pesquisador cuja argumentação, lógica, sensibilidade, criatividade e intuição apontam as dimensões da nova investigação (THERRIEN e NÓBREGA-THERRIEN, 2004, p.4).
\end{abstract}

Nesse sentido, confere-se amplitude e originalidade ao corpo investigativo que se pretende referendar uma vez que possibilita um pressuposto epistemológico consistente à produção do conhecimento atrelado ao atual paradigma da educação científica e suas interfaces metodológicas, didáticas, avaliativas e normativas, as quais em conjunto, pressupõem a compreensão do mundo, interpretação dos fenômenos e suas aplicações cotidianas.

\section{A relação entre o estado da questão e a pesquisa no ensino das ciências: percursos e contribuições.}

De acordo com o panorama catalogado na determinação do estado da questão percebe-se que em essência o conhecimento produzido em função da orientação da ação na perspectiva da elaboração de uma tese que aborde a orientação no contexto da leitura crítica em química, torna-se viável, contundente e original, pois relacionando-a à atual produção do conhecimento, sobretudo em função das habilidades linguístico-cognitivas e ao ensino desenvolvimental percebe-se um hiato sobre esse objeto e o atual estado da questão da orientação da ação. 


\section{Educere "Educare \\ REVISTA DE EdUCACÃ̃o}

Programa de Pós-Graduação em Educação - Universidade Estadual do Oeste do Paraná

Afinal, mesmo com extensa produção em torno dos aspectos teóricoconceituais e linguístico-cognitivos relacionados à orientação da ação, observase uma lacuna em relação às produções que interseccionem a orientação da habilidade da leitura crítica associada ao processo de formação de professores no âmbito da educação científica, logo, apesar de uma série de estudos norteadores e de ampla discussão em torno das categorias adjacentes à proposta, dada essa lacuna é possível compreender a originalidade e novidade em torno desta temática no campo em questão.

Nesse sentido, se reforça a importância da leitura crítica como um pressuposto e uma necessidade formativa, visto que a formação de habilidades linguístico-cognitivas, sobretudo às relacionadas à leitura, é indispensável para o êxito da profissionalização docente e no escopo do estado do conhecimento atual.

Las definiciones de lectura y competencia lectora han evolucionado a lo largo del tiempo de forma paralela a lós cambios sociales, económicos y culturales. El concepto de aprendizaje y, en concreto, el concepto de aprendizaje permanente han ampliado la percepción de la competencia lectora, que ha dejado de contemplarse como una capacidad adquirida únicamente en la infancia, durante los primeros años de la escolarización. En cambio, está considerada como un conjunto expansible de conocimientos, destrezas y estrategias que lós individuos van desarrollando a lo largo de la vida en distintos contextos, a través de la interacción con sus iguales y con la comunidad en general.

(...) Competencia lectora es comprender, utilizar, reflexionar y comprometerse con textos escritos, para alcanzar lospropios objetivos, desarrollar el conocimiento y potencial personales, y participar en la sociedad (OCDE, 2012).

Observando a temática a partir das concepções e orientações curriculares brasileiras, percebe-se seu o grau de pertinência e adequação à realidade educacional vigente, a qual, dentre outras concepções percebe o ensino de Química a partir de um referencial leitor e articulado com o processo da construção de um pensamento crítico atrelado à comunicação e linguagem (BRASIL, 2006). 


\section{Educere Educare \\ RevISTA DE Educacão}

Programa de Pós-Graduação em Educação - Universidade Estadual do Oeste do Paraná

Ademais, partindo da leitura crítica como o estado da questão para além de uma habilidade em si e interseccionando os olhares com as demais competências científicas e habilidades cognitivas, tais como o a formação de um pensamento crítico e autonomia intelectual em ciências/química, a pesquisa, em consonância com a literatura e diversos programas internacionais (OCDE, 2005), pode contribuir para responder lacunas presentes no campo da educação científica brasileira atual, pois, ao se investigar habilidades linguístico-cognitivas como a leitura crítica, dada sua natureza transdisciplinar, torna-se inevitável o desvelamento de outras habilidades relacionadas, uma vez que:

Leer implica establecer relaciones entre el autor, el texto y el lector del texto. Los lectores pueden posicionarse epistemológicamente de diferentes maneras respecto a un texto. (...) el alumnado adopte una postura crítica iniciando una negociación interactiva entre el texto y sus creencias $u$ opiniones para conseguir una interpretación sea lo más consistente y completa posible (OLIVERAS y SANMARTÍ, 2009)

Los textos científicos, aparte de la dificultad propia de la lectura en sí, tienen un modelo de ciencia implícito que dificulta aun más su comprensión. Debido a esto es importante trabajar la lectura y comprensión de textos científicos desde la misma clase de ciencias, (...) entendemos que leer significa comprender, interpretar, analizar y criticar los textos (CASSANY, 2008)

Por fim, ancorando-se na perspectiva do materialismo histórico dialético como suporte teórico da pesquisa, o qual possibilita uma leitura dinâmica entre o objeto e os sujeitos da investigação, objetivam-se por meio da análise das contradições que envolvem a profissionalização docente, as necessidades formativas contemporâneas e o paradigma das competências científicas para o século XXI, elencar a orientação para a leitura crítica no âmbito da didática e da aprendizagem a fim de se proporcionar novos olhares em torno de uma nova configuração dessa base orientadora para o ensino das ciências e a formação dos professores. 


\section{Educere Educare \\ REVISTA DE EdUCACÃ̃}

Programa de Pós-Graduação em Educação - Universidade Estadual do Oeste do Paraná

\section{REFERÊNCIAS:}

BELL, Judith. Doing Your Research Project: A guide for first-time researchers. McGraw-Hill Education (UK), 2014.

DA SILVA FAQUIM, Juliana Pereira; PUENTES, Roberto Valdés. Diferentes concepções de ensino: da didática do estímulo à didática do desenvolvimento. e-RAC, v. 1, n. 1, 2013.

GALPERIN, P. Ya. Sobre método y formación por etapas de las acciones intelectuales.: Antología de las edades. Sobre método y formación por etapas de las acciones intelectuales.: Antología de las edades., 1986.

LAROCCA, Priscila; ROSSO, Ademir José; DE SOUZA, Audrey Pietrobelli. A formulação dos objetivos de pesquisa na pós-graduação em Educação: uma discussão necessária. Revista Brasileira de Pós-Graduação, v. 2, n. 3, 2005.

LIBÂNEO, José Carlos; FREITAS, R. A. M. M. Vasily Vasilyevich Davydov: a escola e a formação do pensamento teórico-científico.Ensino desenvolvimental: vida, pensamento e obra dos principais representantes russos. Uberlândia: EDUFU, p. 315-350, 2013.

LEONTIEV, Alexei Nikolaevich. Sobre o desenvolvimento histórico da consciência. $\mathbf{O}$ desenvolvimento do psiquismo, 1978.

LURIA, Alexander Romanovich; LEONTIEV, Alexis. Psicologia y pedagogia. Ediciones Akal, 2004.

MARTINS, Onilza Borges; MOSER, Alvino. Conceito de mediação em Vygotsky, Leontiev e Wertsch. Revista Intersaberes, v. 7, n. 13, p. 8-28, 2012.

NÓBREGA-THERRIEN, Sílvia Maria; THERRIEN, Jacques. Trabalhos científicos e o estado da questão. Estudos em avaliação educacional, v. 15, n. 30, p. 5-16, 2004. 


\section{Educere Educare \\ REVISTA DE EDUCACÃo}

Programa de Pós-Graduação em Educação - Universidade Estadual do Oeste do Paraná

NÚÑEZ, Isauro Beltrán. Vygotsky, Leontiev, Galperin. Formação de conceitos e principios didáticos, 2009.

PUENTES, Roberto V.; LONGAREZI, Andréa M. Escola e didática desenvolvimental: seu campo conceitual na tradição da teoria históricocultural. Educação em revista, v. 29, n. 1, p. 247-271, 2013.

RAMOS VOSGERAU, Dilmeire Sant'Anna; ROMANOWSKI, Joana Paulin. Estudos de revisão: implicações conceituais e metodológicas. Revista Diálogo Educacional, v. 14, n. 41, 2014.

SILVEIRA, Clarice Santiago; NÓBREGA-THERRIEN, Sílvia Maria. Estudos sobre pesquisa e formação de professores da Educação Básica: a elaboração do Estado da Questão. Revista Educação em Questão, v. 41, n. 27, 2011.

TALÍZINA, N. F. La formación de los conceptos matemáticos. La formación de las habilidades del pensamiento matemático, p. 21-39, 2001.

TOASSA, Gisele. "Behind consciousness, there is life": the LeontievVygotsky theoretical departure in the network of vygotskian scholars. Educação \& Sociedade, v. 37, n. 135, p. 445-46

\footnotetext{
${ }^{\mathrm{i}}$ Legenda das siglas do quadro 1 :

UERR: Universidade Estadual de Roraima; IFES:Instituto Federal do Espírito Santo; UNIUBE: Universidade de Uberaba; UFRN:Universidade Federal do Rio Grande do Norte; UTFPR: Universidade Tecnológica Federal do Paraná

ii Legenda das siglas do quadro 2:

UFU: Universidade Federal de Uberlândia; USP: Universidade de São Paulo; UFRPE Universidade Federal Rural de Pernambuco; UFPB: Universidade Federal da Paraíba; UFRN: Universidade Federal do Rio Grande do Norte.
}

Recebido em: 07/02/2018 Aprovado em: 26/07/2018 